\title{
Organic molecules in the spectral line survey of Orion KL with the Odin Satellite from $486-492 \mathrm{GHz}$ and $541-577 \mathrm{GHz}$
}

\author{
N. Koning ${ }^{1}$, S. Kwok ${ }^{1,2}$, P. Bernath ${ }^{3}$, Å. Hjalmarson ${ }^{4}$, and \\ H. Olofsson ${ }^{4}$ \\ ${ }^{1}$ Department of Physics and Astronomy, University of Calgary, \\ Calgary, AB, T2N 1N4, Canada \\ email: nkoning@iras.ucalgary.ca, sunkwok@hku.hk \\ ${ }^{2}$ Department of Physics, University of Hong Kong, Pokfulam Road, Hong Kong, China \\ ${ }^{3}$ Department of Physics, University of Waterloo, Waterloo, Ontario, N2L 3G1, Canada \\ ${ }^{4}$ Onsala Space Observatory, Chalmers University of Technology, 439 92, Onsala, Sweden
}

\begin{abstract}
A spectral line survey of Orion KL has been performed over the frequency range of 486-492 GHz and 541-577 GHz using the Odin satellite. Over 1000 lines have been identified from 40 different molecular species, including several organic compounds such as methyl cyanide $\left(\mathrm{CH}_{3} \mathrm{CN}\right)$, methanol $\left(\mathrm{CH}_{3} \mathrm{OH},{ }^{13} \mathrm{CH}_{3} \mathrm{OH}\right)$, and dimethyl ether $\left(\mathrm{CH}_{3} \mathrm{OCH}_{3}\right)$.
\end{abstract}

Keywords. Astrochemistry, submillimeter

\section{Introduction}

Although there have been over 20 ground-based spectral surveys of the Orion Kleinmann-Low Nebula, observations between $500 \mathrm{GHz}$ and $600 \mathrm{GHz}$ have not been possible from the ground due to atmospheric absorption by $\mathrm{H}_{2} \mathrm{O}$ and $\mathrm{O}_{2}$. The Odin Satellite (Hjalmarson et al. 2003) is capable of observing frequencies in the range of 486-504 GHz and $541-581 \mathrm{GHz}$ via 4 tunable receivers. Odin therefore provides a great opportunity to observe this unexplored region of Orion KL's spectrum.

\section{Observations and analysis}

Odin performed a spectral line survey of the Orion KL region over a 1.5 year period from February 2004 to November 2005, spanning 1100 orbits. All four tunable receivers were used to observe between 486-492 GHz and 541-577 GHz. The observations were made with a beam size of $2.1^{\prime}$ (at $557 \mathrm{GHz}$ ) in position switching mode where the entire telescope physically moved $15^{\prime}$ between the target and an off position with a cycle period of 1 minute.

The data were processed and analyzed in parallel by the Space Astronomy Laboratory (SAL) in Calgary and the Onsala Space Observatory in Sweden. A part of the surveyed spectrum between 541-577 GHz is shown in Figure 3. The JPL Molecular Spectroscopy catalogue (Pickett et al. 1998) and the Cologne Database for Molecular Spectroscopy (CDMS, Müller et al. 2005) were used in the line identification. In the case of methanol, a separate list of transitions supplied by Eric Herbst was used. Gaussian profiles were fit to each observed feature using a least squares algorithm. In the case where more than one transition contributed to a single feature, special routines were used to fit the sum of several profiles. In some situations the number of blended lines was excessive and no fits were attempted due to substantial ambiguities. 


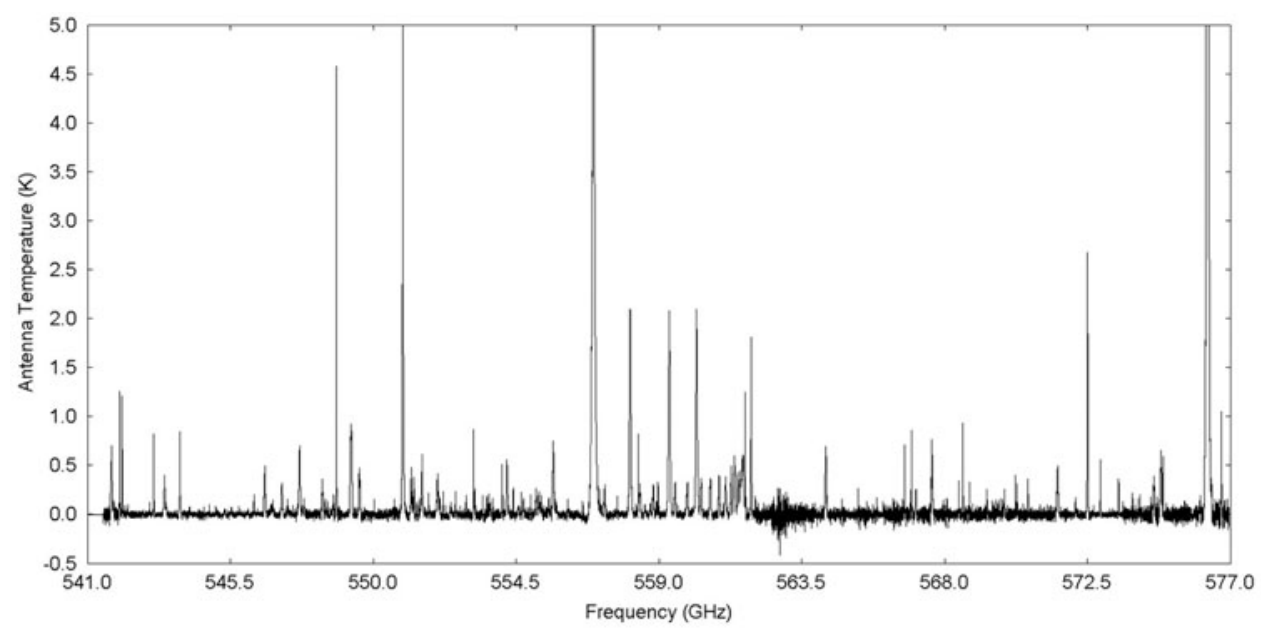

Figure 1. Spectrum of Orion KL from 541-577 GHz observed with the Odin satellite. Several organic molecules are present, including methanol, methyl cyanide, dimethyl ether, formaldehyde, thio-formaldehyde, thioformyl cation and hydrogen isocyanide. The strongest lines in this spectrum are $\mathrm{CO} J=5-4(576.27 \mathrm{GHz}),{ }^{13} \mathrm{CO} J=5-4(550.93 \mathrm{GHz}), \mathrm{C}^{18} \mathrm{O} J=5-4$ $(548.83 \mathrm{GHz})$, and $\mathrm{H}_{2} \mathrm{O} 1_{10}-1_{01}(556.94 \mathrm{GHz})$.

\section{Organic Molecules}

Seven organic molecules were detected in this survey: methanol $\left(\mathrm{CH}_{3} \mathrm{OH},{ }^{13} \mathrm{CH}_{3} \mathrm{OH}\right)$, methyl cyanide $\left(\mathrm{CH}_{3} \mathrm{CN}\right)$, dimethyl ether $\left(\mathrm{CH}_{3} \mathrm{OCH}_{3}\right)$, formaldehyde $\left(\mathrm{H}_{2} \mathrm{CO}, \mathrm{H}_{2}^{13} \mathrm{CO}\right.$, $\mathrm{HDCO})$, thio-formaldehyde $\left(\mathrm{H}_{2} \mathrm{CS}\right)$, thioformyl cation $\left(\mathrm{HCS}^{+}\right)$, and hydrogen isocyanide (HNC). Methanol dominates the entire survey with 83 detected lines making it ideal for rotation diagram analysis. Both $\mathrm{A}$ and $\mathrm{E}$ species were detected, with a ratio of $\sim 3: 1$ for $\mathrm{CH}_{3} \mathrm{OH}$ and $2: 1$ for ${ }^{13} \mathrm{CH}_{3} \mathrm{OH}$. The temperature determined for methanol is $181 \mathrm{~K}$. ${ }^{13} \mathrm{CH}_{3} \mathrm{OH}$ had enough lines to warrant a rotation diagram of its own, with a derived temperature of $110 \mathrm{~K}$. Ninety-eight lines of methyl cyanide were detected, but due to overcrowding among fine structure lines, only 9 could be profiled. Using these lines, a rotation diagram was constructed yielding a highly uncertain temperature of $62 \mathrm{~K}$ and a column density of $5.7 \times 10^{17} \mathrm{~cm}^{-2}$. Dimethyl ether had 413 transitions detected, although due to the AA, AE, EE and EA symmetries these were almost all fine structure lines and not resolvable. As such, too few lines could be profiled in order to create a reliable rotation diagram. Only three transitions of formaldehyde were detected and only a single transition of both $\mathrm{HDCO}$ and $\mathrm{H}_{2}^{13} \mathrm{CO}$ were detected. The thioformyl cation was also detected only once. Odin observed 6 transitions of thioformaldehyde, enough to allow for a rotation diagram to be constructed. The derived temperature and column density is $124 \mathrm{~K}$ and $4.3 \times 10^{13} \mathrm{~cm}^{-2}$, respectively. Hydrogen isocyanide was only detected once at $543.9 \mathrm{GHz}$, and is highly blended with several $\mathrm{CH}_{3} \mathrm{OCH}_{3}$ lines and one unidentified line.

\section{References}

Hjalmarson, A., Frisk, U., Olberg, M., Bergman, P., Bernath, P., et al. 2003, A $\dot{E} A$ (Letters), 402, L39

Müller, H. S.P., Schlöder, F., Stutzki, J., \& Winnewisser, G. 2005, J. Molecular Struct., 742, 215

Pickett, H. M., Poynter, R. L., Cohen, E. A., Delitsky, M. L., Pearson, J. C., \& Muller, H. S. P. 1998, J. Wuant. Spectrosc. \& Rad. Transfer, 60 